Biomath Communications

http://www.biomathforum.org/biomath/index.php/conference Biomath Forum

\title{
In Memoriam: A Tribute to Our Colleague and Friend, Asen L. Dontchev
}

\author{
Mikhail I. Krastanov ${ }^{1}$, Svetoslav M. Markov², Vladimir M. Veliov ${ }^{3}$ \\ ${ }^{1}$ Faculty of Mathematics and Informatics, \\ Sofia University "St. Kliment Ohridski", Bulgaria \\ krastanov@fmi.uni-sofia.bg \\ ${ }^{2}$ Institute of Mathematics and Informatics, \\ Bulgarian Academy of Sciences, Bulgaria \\ smarkov@bio.bas.bg \\ ${ }^{3}$ Institute of Statistics and Mathematical Methods in Economics, \\ Vienna University of Technology, Austria \\ vladimir.veliov@tuwien.ac.at
}

Asen Lyubomirov Dontchev, an internationally renowned mathematician in the fields of variational analysis, approximation theory, optimization and control theory, passed away in Ann Arbor, MI, on September 16, 2021.

Asen was born on June 19, 1948, in the city of Pleven, Bulgaria. After completing his high school education, showing extraordinary mathematical talent, he was accepted in the Warsaw University of Technology, where he received M.Sc. (in 1971) and Ph.D. in Control Sciences (in 1974). After his graduation he joined the Department of Operations Research of the Institute of Mathematics and Informatics of the Bulgarian Academy of Sciences. In 1987 Asen became Doctor of Mathematical Sciences and Full Professor. Since 1990 he has been Associate Editor at Mathematical Reviews and since 2000 - Adjunct Professor at the University of Michigan. From 2007 till 2009 he was acting as a Program Director of the Analysis Program, DMS of the US National Science Foundation.

Copyright: (C) 2021 Mikhail I. Krastanov, Svetoslav M. Markov, Vladimir M. Veliov. This article is distributed under the terms of the Creative Commons Attribution License 4.0, which permits unrestricted use, distribution, and reproduction in any medium, provided the original author and source are credited. Published: November 9, 2021, Updated: June 2, 2022

Citation: Mikhail I. Krastanov, Svetoslav M. Markov, Vladimir M. Veliov, In Memoriam: A Tribute to Our Colleague and Friend, Asen L. Dontchev, Biomath Communications 8 (2021), 2111091, https://doi.org/10.11145/bmc.2021.11.091 


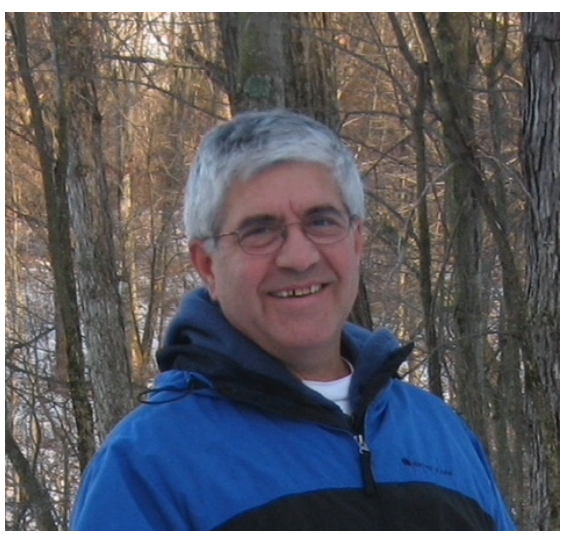

Asen Dontchev 1948-2021

Asen Dontchev has more than 180 publications, including three books (published by Springer, one of which was translated into Russian) and three textbooks [1, 2, 3, 4].

He was member of the editorial boards of the following international journals: Serdica Mathematical Journal, Computational Optimization and Applications, SIAM Journal on Optimization, Journal of Dynamical and Control Systems, SIAM Journal on Control and Optimization, Open Journal of Applied Mathematics, Journal of Industrial and Managerial Optimization, Mathematica Balkanica, Journal of Mathematical Analysis and Applications, Journal of Optimization Theory and Applications.

Asen has made several important contributions in the fields of variational analysis, optimization and control. In a series of works on singularly perturbed optimal control problems he introduced a new concept of limit problem, which goes beyond the usual formal application of the classical Tikhonov theorem. In collaboration with William W. Hager, Asen developed a powerful approach for studying stability properties of generalized equations. The ideas of this approach have important applications in the stability analysis and approximation theory for optimization problems, including optimal control. This line of research was extended and complemented by Asen's works on inverse mappings and implicit function theorems for set-valued mappings, which culminated in the book [3] by him and Terry Rockafellar.

This book presents several fundamental concepts and results in variational analysis: various regularity properties of set-valued mappings, implicit function theorems, radius of regularity, and applications in numerical analysis. One of these concepts, the so called metric subregularity, introduced and systematically studied by Asen and his collaborators, became an important instrument in the approximation theory for optimization problems. Asen's last few years were devoted to justification and improvement of the method of model predictive control, which is an important tool for 
process control in industry, aerospace engineering and power electronics.

Throughout his career, Asen was a mentor of several generations of mathematicians and had supervised number of Ph.D. students. He was an excellent lecturer and had a remarkable ability to disseminate his knowledge and to inspire young researchers. Due to his openness and friendliness, he had a large number of scientific contacts and visits in universities all over the world.

Moreover, Asen loved the outdoors, and he was an avid fisherman. The place he was happiest and most relaxed was being on the water in his boat. He was extremely proud of how well his children were doing, and he was an enthusiastic grandfather.

Asen is survived by his wife Dora, daughter Mira, son Kiko, and three grandchildren. He has many friends, students and collaborators, and is deeply missed by everyone who knows him.

\section{References}

[1] A.L Dontchev. Perturbations, approximations and sensitivity analysis of optimal control systems. Lecture Notes in Control and Information Sciences, 52. Springer-Verlag, Berlin, 1983.

[2] A.L Dontchev and T. Zolezzi. Well-posed optimization problems. Springer, New York, 1993.

[3] A.L. Dontchev, R.T. Rockafellar. Implicit Functions and Solution Mappings: A View from Variational Analysis. Second edition. Springer, New York, 2014.

[4] A.L Dontchev. Lectures on Variational Analysis. Applied Mathematical Sciences, Volume 205. Springer, Cham, 2021. 